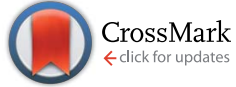

Cite this: RSC Adv., 2016, 6, 17125

Received 18th January 2016

Accepted 3rd February 2016

DOI: $10.1039 / \mathrm{c} 6 \mathrm{ra01541g}$

www.rsc.org/advances

\section{Organic/inorganic epitaxy: commensurate epitaxial growth of truxenone on $\mathrm{Cu}(111) \dagger$}

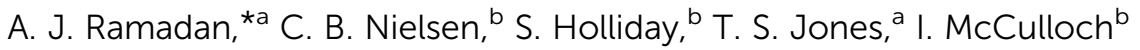 \\ and L. A. Rochford*a
}

The growth of monolayers of truxenone on $\mathrm{Cu}$ (111) is investigated using scanning tunneling microscopy (STM) and low energy electron diffraction (LEED). Two distinct molecular packing motifs are observed that exist individually at low and high coverage, and coexist at intermediate states. In each case a commensurate epitaxial relationship between the molecular surface mesh and the substrate is observed.
The interface between organic molecules and metallic surfaces is integral to the performance of organic optoelectronic devices. ${ }^{1}$ Considerable efforts have been focussed on the synthesis and processing of novel donor and acceptor materials for high performance devices. ${ }^{2-6}$ Despite this, there is little understanding of the fundamental behaviour of these molecules at relevant metallic surfaces. Of particular interest is the growth behaviour at the earliest stages of thin film formation. ${ }^{7}$ Epitaxial growth has the potential to create single-crystalline films which exhibit very low defect concentrations and few carrier trapping sites. Incommensurate epitaxial relationships have been shown to be relatively common between large organic molecules and metal surfaces. ${ }^{8-11}$ However preparing and characterising commensurate epitaxial organic/metal structures represents only a tiny portion of the literature. ${ }^{12-15}$ Preparing these kinds of structures allows the translational symmetry of the substrate to be preserved in the organic structure templated on top. As structure-property relationships are important in organic semiconductor systems structural control is a vital step in ensuring optimal performance in electronic devices. ${ }^{16}$ The 'bottom-up' approach we present is a key stage in understanding the fundamental film formation processes at work in device growth.

The truxenones family of small molecule semiconductors exhibit three-fold symmetry, have relatively stabilised lowest unoccupied molecular orbital (LUMO) energy levels, independently conjugated branches from the core and have been shown to exhibit promising properties as electron acceptors in organic solar cells. ${ }^{17,18}$ The parent truxenone (shown in Fig. 1) has been shown to form supramolecular assemblies in the monolayer regime on $\mathrm{Au}$ (111)/Mica and highly ordered pyrolytic graphite

${ }^{a}$ Department of Chemistry, University of Warwick, Gibbet Hill Road, Coventry, CV4 7AL, UK.E-mail: a.ramadan@warwick.ac.uk; luke.rochford@warwick.ac.uk

${ }^{b}$ Department of Chemistry, Imperial College London, South Kensington, SW7 2AZ, UK $\uparrow$ Electronic supplementary information (ESI) available: STM images, simulated LEED patterns, FFT images. See DOI: 10.1039/c6ra01541g
(HOPG) surfaces. Although high resolution imaging has been employed the nature of their surface structure remains ambiguous. ${ }^{19,20}$ Herein we study the epitaxial growth of truxenone on $\mathrm{Cu}$ (111) using low energy electron diffraction (LEED) and scanning tunnelling microscopy (STM). We demonstrate two unique epitaxial surface meshes which are dependent on the coverage of truxenone molecules.

Truxenone was synthesised following a literature procedure. ${ }^{21}$ This was then triply purified by thermal gradient sublimation before degassing $20{ }^{\circ} \mathrm{C}$ below the evaporation temperature for several days. All characterisation was carried out at ambient temperature in a custom-built multi-chamber ultra-high vacuum (UHV) system with a base pressure better than $3 \times 10^{-10}$ mbar. Standard low-temperature effusion cells (Karl Eberl) were used at $150{ }^{\circ} \mathrm{C}$ cell temperature to grow truxenone layers. STM images were recorded with an STM/AFM (Omicron) operated in constant current mode using electrochemically etched polycrystalline tungsten tips. Applied voltages and tunnelling currents are indicated in figure captions. LEED patterns were collected with a SPECTALEED (Omicron) rear-view MCP-LEED with nano-amp primary beam current.

Images of these diffraction patterns were captured using a digital CCD camera interfaced to a personal computer. A single crystal $\mathrm{Cu}$ (111) substrate (Surface Preparation Lab, NL cutting accuracy $0.1^{\circ}$ ) was prepared in vacuum by repeated cycles of argon ion sputtering and annealing $\left(\mathrm{Ar}^{+}\right.$energy $1.5 \mathrm{keV}$

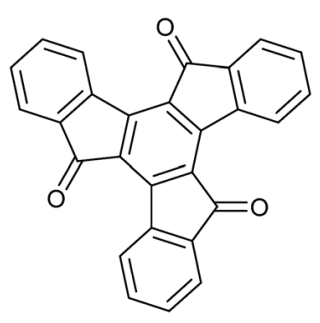

Fig. 1 Molecular structure of truxenone. 
temperature $550{ }^{\circ} \mathrm{C}$ ). Truxenone monolayers were produced by evaporation onto a clean $\mathrm{Cu}$ (111) surface, initially monitored using post growth LEED with increasing deposition time. A diffuse diffraction ring was first observed at low $(20 \mathrm{eV})$ electron beam energy but with continued growth this was replaced by a well-defined spot pattern after 4 minutes of truxenone growth at $150{ }^{\circ} \mathrm{C}$ sublimation temperature. In this work two additional samples will be discussed; truxenone growth for 5.5 minutes and 7 minutes at $150{ }^{\circ} \mathrm{C}$ sublimation temperature. After the 5.5 and 7 minute growths a short annealing procedure $\left(190{ }^{\circ} \mathrm{C}\right.$ for 10 minutes) was employed to produce sharp LEED patterns. This annealing step has not been employed in published photovoltaic devices based on truxenone. ${ }^{17,18}$

After 4 minutes of growth the resulting layers were investigated by STM and LEED and the data are shown in Fig. 2a and d. STM images show that the molecules appear triangular and are oriented in a honeycomb structure defined by a rhombic twomolecule unit cell $\left(r_{1}=r_{2}=2.04 \mathrm{~nm}\right.$ from LEED, $r_{1}=$ $2.07 \mathrm{~nm} \pm 0.1 \mathrm{~nm}$ from STM) similar to that observed in the work on $\mathrm{Au}$ (111)/Mica of Chen and co-workers. ${ }^{20}$ The LEED pattern collected from this surface shows a primitive $(8 \times 8)$ pattern indicative of commensurate epitaxial order between the truxenone molecules and the $\mathrm{Cu}$ (111). No annealing procedure was required to produce a sharp LEED pattern, however where annealing was undertaken $\left(190{ }^{\circ} \mathrm{C}\right.$ for 10 minutes $)$ with
4 minute growth time the LEED pattern remained unchanged. This kind of ordering not only dictates that the primitive lattice vectors of the truxenone layer are integer multiples of the substrate primitives, but also that the translational symmetry of the surface reflects that of the substrate. It is therefore unsurprising that the spots of the truxenone LEED pattern align with the $(1 \times 1)$ beams of the $\mathrm{Cu}(111)$ surface - in both reciprocal and real space the primitives of the $\mathrm{Cu}$ and truxenone layers are aligned (see ESI $\dagger$ ). LEED patterns (for both commensurate structures) collected away from normal incidence are presented in the ESI $\dagger$ for easy identification of the overlayer periodicity.

The surface structure alters dramatically when truxenone growth time is increased from 4 minutes to 5.5 minutes and the surface is briefly annealed. This can be seen in the STM image in Fig. 2b which shows a modification in the packing of the molecules, areas of honeycomb ordering are still present but surrounded by areas of hexagonally close packed molecules. The additional molecules are accommodated into the structure through a change in the molecular packing at the borders of honeycomb packed regions. In controlled annealing experiments (4 minutes growth time) no change to the surface structure was observed by STM or LEED suggesting that additional molecules are required to form the closed packed hexagonal structure. A complete modification of the supramolecular structure is obvious after the longest growth (7 minutes)

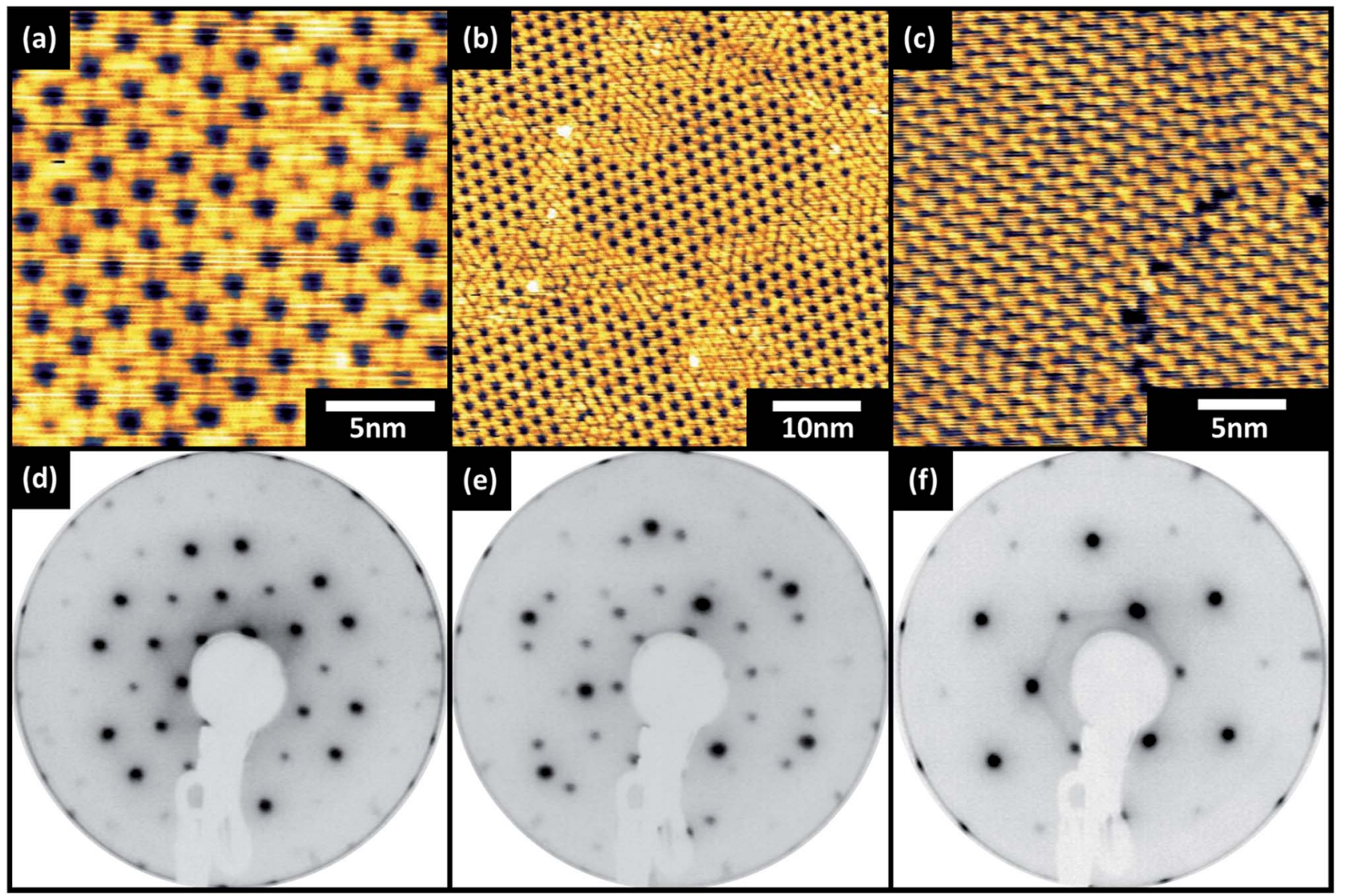

Fig. 2 STM images $\left(V_{S}=-1.25 \mathrm{~V}, I_{T}=100 \mathrm{pA}\right)$ of $\mathrm{Cu}$ (111) with truxenone layers grown for (a) 4 minutes, (b) 5.5 minutes, (c) 7 minutes and corresponding LEED images captured at $20 \mathrm{eV}$ of (d) 4 minutes, (e) 5.5 minutes and (f) 7 minutes. 
and short anneal. Fig. 2c shows that the surface structure is defined by a single molecule unit cell $\left(r_{1}=r_{2}=1.27 \mathrm{~nm}\right.$ from LEED, $r_{1}=1.25 \mathrm{~nm} \pm 0.1 \mathrm{~nm}$ from STM) which produces hexagonal close packing. This change in molecular packing arrangement is reflected in LEED patterns captured for both the intermediation and longest growths. Fig. $2 \mathrm{f}$ (corresponding to 7 minute growth $)$ shows a $\mathrm{p}(5 \times 5)$ pattern which, like the pattern observed for the shortest growth time, is indicative of a commensurate epitaxial structure. The change in the pattern from a $\mathrm{p}(8 \times 8)$ to a $\mathrm{p}(5 \times 5)$ also confirms the observation in the STM images that the molecules are closer together in the unit cell of this surface. The LEED pattern of the intermediate (5.5 minute) growth of truxenone is a combination of the $\mathrm{p}(5 \times$ $5)$ and the $\mathrm{p}(8 \times 8)$ structures observed for the long and short growths. The concurrent observation of these patterns shows that the combination of the two packing arrangements observed in the STM images is also reflected on the longer length scales probed in LEED. Larger areas of the three systems were imaged and the corresponding STM images can be seen in Fig. 3. The open hexagonal $\mathrm{p}(8 \times 8)$ structure forms domains of approximately $50 \mathrm{~nm}$ in size, although each domain has translational symmetry. As longer growth and a short annealing procedure is undertaken the close packed $\mathrm{p}(5 \times 5)$ regions define the edges of the $\mathrm{p}(8 \times 8)$ domains. When coverage increases further (and after annealing) the $\mathrm{p}(5 \times 5)$ regions extend to possess comparable dimensions to the starting $\mathrm{p}(8 \times$ 8 ) domains. Small areas of $\mathrm{p}(8 \times 8)$ order can be identified suggesting that the interconversion process may not be complete, but only a single structure was detected in the LEED.

It has been previously reported that when immobilised on a surface the truxenone molecule forms two enantiomers and that it may be these which are responsible for the different packing. ${ }^{20}$ Discrimination between the two enantiomers is not possible from our STM images and although theoretical calculations have been previously employed the authors concluded that the energetic difference between enantiomorphous and enantiopure molecule pairs and assemblies are negligible when compared to the molecule-substrate interaction. Our data demonstrate that the supramolecular structures present on $\mathrm{Cu}$ (111) can accommodate both enantiomers. More explicitly, if intermolecular interactions between enantiomers pairs $(\mathrm{R}+\mathrm{R}, \mathrm{L}$ $+\mathrm{L}$ and $\mathrm{L}+\mathrm{R}$ ) were significantly different energetically, multiple similar unit cells or mirrored domains would be expected to be present. As STM images and, more importantly, LEED patterns only show a single domain sharing lattice directions with the $\mathrm{Cu}$ (111) mesh this is precluded. Discriminating whether this is due to the segregation of each enantiomer into discrete domains or due to the fact the packing arrangement of the molecules is not sensitive to the 'handedness' of each surface enantiomer is beyond the capabilities of our combined STM and LEED measurements. Additionally, the adsorption configuration (in terms of molecular orientation and binding sites) could only be unambiguously determined using synchrotron based techniques such as normal incidence X-ray standing wave (NIXSW), and photoelectron diffraction (PhD). In the absence of this data a unique solution to the absolute surface structure cannot be presented, although the surface mesh extracted from LEED and STM remains valid.

In all cases we observe no mirror symmetry related structures i.e. domains that are tilted with respect to the underlying $\mathrm{Cu}$ (111) surface mesh. This is presumably due to direct alignment of the truxenone unit cell with the close packed directions of the substrate.

The existence of a commensurate epitaxial relationship between an organic molecule and inorganic surface is, in itself, a rarity in the field. The presence of two distinct commensurate epitaxial relationships at different molecular coverages is highly unusual and to the authors' knowledge previously unreported for any organic semiconducting molecule. The growth mode of organic semiconductors has been shown to have a direct correlation with the electronic and physical properties of resulting thin films and the existence of epitaxy presents an alternative method through which the growth mode may be controllable. ${ }^{22-25}$ In particular the potential to grow different structures, both as a result of commensurate epitaxy, presents a unique opportunity to produce two equally high quality films which would likely have two different sets of physical properties.

In summary this work has shown the commensurate epitaxial growth of truxenone on $\mathrm{Cu}$ (111). Two distinct surface
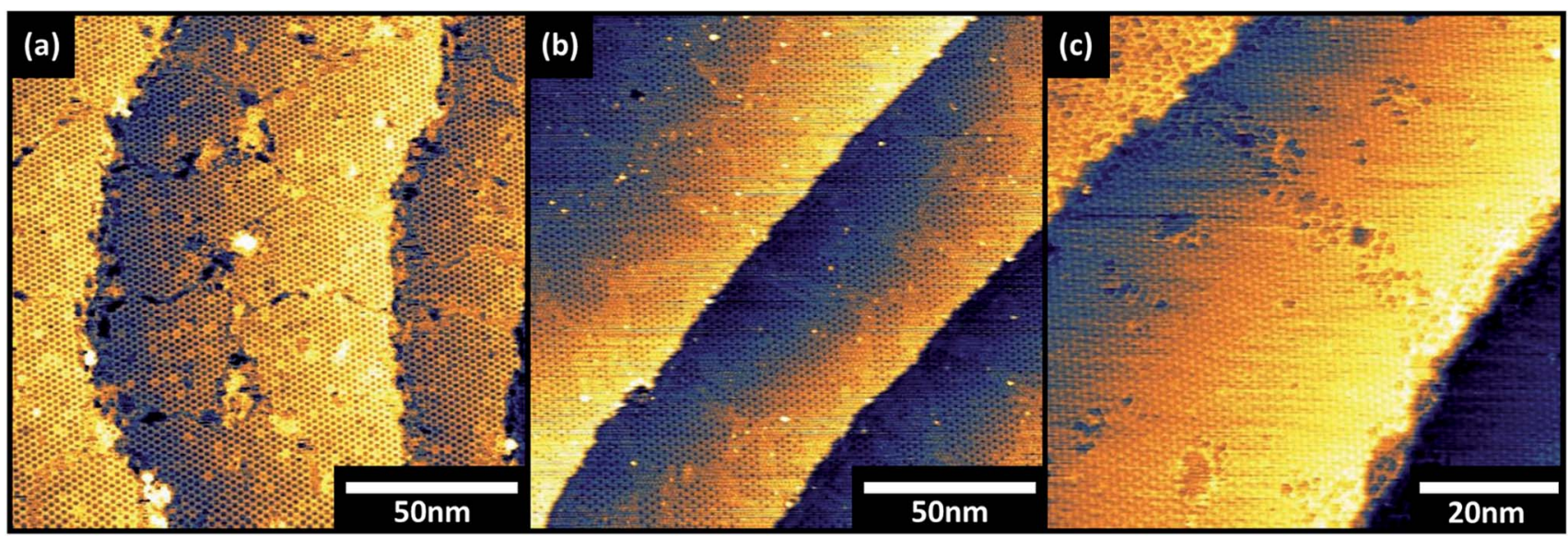

Fig. 3 STM images $\left(V_{S}=-1.25 \mathrm{~V}, I_{T}=100 \mathrm{pA}\right)$ of truxenone layers grown on Cu (111) for (a) 4 minutes, (b) 5.5 minutes and (c) 7 minutes. 
meshes have been observed for different coverages of truxenone, one hexagonally closed packed, $\mathrm{p}(5 \times 5)$, and one honeycomb structure, $\mathrm{p}(8 \times 8)$, which co-exist at intermediate coverages. This is the first demonstration of two unique commensurate epitaxial structures of a large conjugated molecule on a metallic surface.

\section{Acknowledgements}

AJR, TSJ and LAR acknowledge support from the Engineering and Physical Sciences Research Council [Grant Number EP/ H021388/1]. CN, SH and IM acknowledge support from the following funding bodies: EPSRC Project EP/G037515/1, EC FP7 Project SC2 (610115), EC FP7 Project ArtESun (604397), and EC FP7 POLYMED (612538). The raw data associated with this article can be accessed at the following DOI: 10.6084/ m9.figshare. 2074360

\section{Notes and references}

1 A. Hinderhofer and F. Schreiber, ChemPhysChem, 2012, 13, 628-643.

2 Y. Liu, J. Zhao, Z. Li, C. Mu, W. Ma, H. Hu, K. Jiang, H. Lin, H. Ade, H. Yan, K. H. Ong, S. L. Lim, J. Li, H. K. Wong, H. S. Tan, T. T. Lin, L. C. H. Moh, J. C. de Mello, Z. K. Chen, Y. Liu, J. Zhao, Z. Li, C. Mu, W. Ma, H. Hu, K. Jiang, H. Lin, H. Ade and H. Yan, Nat. Commun., 2014, 5, 1-8.

3 C. B. Nielsen, R. S. Ashraf, N. D. Treat, B. C. Schroeder, J. E. Donaghey, A. J. P. White, N. Stingelin and I. McCulloch, Adv. Mater., 2015, 27, 948-953.

4 Y. Zhong, M. T. Trinh, R. Chen, G. E. Purdum, P. P. Khlyabich, M. Sezen, S. Oh, H. Zhu, B. Fowler, B. Zhang, W. Wang, C.-Y. Nam, M. Y. Sfeir, C. T. Black, M. L. Steigerwald, Y.-L. Loo, F. Ng, X.-Y. Zhu and C. Nuckolls, Nat. Commun., 2015, 6, 8242.

5 S. Holliday, R. S. Ashraf, C. B. Nielsen, M. Kirkus, J. A. Röhr, C.-H. Tan, E. Collado-Fregoso, A.-C. Knall, J. R. Durrant, J. Nelson and I. McCulloch, J. Am. Chem. Soc., 2015, 137, 898-904.

6 K. Cnops, B. P. Rand, D. Cheyns, B. Verreet, M. A. Empl and P. Heremans, Nat. Commun., 2014, 5, 3406.
7 F. Schreiber, Phys. Status Solidi A, 2004, 201, 1037-1054.

8 B. Stadtmüller, I. Kröger, F. Reinert and C. Kumpf, Phys. Rev. B: Condens. Matter Mater. Phys., 2011, 83, 85416.

9 L. A. Rochford, I. Hancox and T. S. Jones, Surf. Sci., 2014, 628, 62-65.

10 C. Stadler, S. Hansen, I. Kröger, C. Kumpf and E. Umbach, Nat. Phys., 2009, 5, 153-158.

11 I. Kröger, B. Stadtmüller, C. Stadler, J. Ziroff, M. Kochler, A. Stahl, F. Pollinger, T. L. Lee, J. Zegenhagen, F. Reinert and C. Kumpf, New J. Phys., 2010, 12, 083038.

12 C. Seidel, R. Ellerbrake, L. Gross and H. Fuchs, Phys. Rev. B: Condens. Matter Mater. Phys., 2001, 64, 195418.

13 M. Kiel, K. Duncker, C. Hagendorf and W. Widdra, Phys. Rev. B: Condens. Matter Mater. Phys., 2007, 75, 195439.

14 K. Duncker, M. Kiel, A. Höfer and W. Widdra, Phys. Rev. B: Condens. Matter Mater. Phys., 2008, 77, 155423.

15 Q. Chen, T. Rada, A. McDowall and N. V. Richardson, Chem. Mater., 2002, 14, 743-749.

16 J. E. Anthony, Nat. Mater., 2014, 13, 773-775.

17 C. B. Nielsen, E. Voroshazi, S. Holliday, K. Cnops, B. P. Rand and I. McCulloch, J. Mater. Chem. A, 2013, 1, 73-76.

18 C. B. Nielsen, E. Voroshazi, S. Holliday, K. Cnops, D. Cheyns and I. McCulloch, J. Mater. Chem. A, 2014, 2, 12348-12354.

19 Z.-Y. Yang, Y. Tao, T. Chen, H.-J. Yan and Z.-X. Wang, Phys. Chem. Chem. Phys., 2013, 15, 2105.

20 F. Chen, Z. Hu, Y. Ji, A. Zhao, B. Wang, J. Yang and J. G. Hou, Phys. Chem. Chem. Phys., 2012, 14, 3980.

21 E. V. Dehmlow and T. Kelle, Synth. Commun., 1997, 27, 20212031.

22 K. Vasseur, K. Broch, A. Ayzner, B. P. Rand, D. Cheyns, C. Frank, F. Schreiber, M. F. Toney, L. Froyen and P. Heremans, ACS Appl. Mater. Interfaces, 2013, 5, 8505-8515.

23 B. P. Rand, D. Cheyns, K. Vasseur, N. C. Giebink, S. Mothy, Y. Yi, V. Coropceanu, D. Beljonne, J. Cornil, J. L. Brédas and J. Genoe, Adv. Funct. Mater., 2012, 22, 2987-2995.

24 P. Sullivan, T. S. Jones, A. J. Ferguson and S. Heutz, Appl. Phys. Lett., 2007, 91, 233114.

25 M. Gruber, M. Rawolle, J. Wagner, D. Magerl, U. Hörmann, J. Perlich, S. V. Roth, A. Opitz, F. Schreiber, P. MüllerBuschbaum and W. Brütting, Adv. Energy Mater., 2013, 3, 1075-1083. 